\title{
Unified Theory of Gravity and Electromagnetic Field with Problem in Reissner-Nordstrom and Kerr-Newman Solution
}

\author{
Sangwha-Yi* \\ Department of Math, Taejon University 300-716, South Korea
}

*Corresponding Author: Sangwha-Yi, Department of Math, Taejon University 300-716, South Korea

\begin{abstract}
Solutions of unified theory equations of gravity and electromagnetism satisfy Einstein-Maxwell equation. Hence, solutions of the unified theory is Reissner-Nordstrom solution in vacuum. We found in revised Einstein gravity tensor equation, the condition is satisfied by 2-order contravariant metric tensor two times product. In theory, the problem that the special Ricci tensor have two value term is solved by two orientation of rotating (right hand and left hand).
\end{abstract}

Keywords: General relativity theory, Unified Theory; 2-order contravariant metric tensor two times product.

PACS Number: 04,04.90.+e,41.12

\section{INTRODUCTION}

This theory's aim is that we discover the revised Einstein gravity equation had Reissner-Nordstrom solution in vacuum.

First, we can think the following formula (the revised Einstein gravity equation).

$R_{\mu \nu}-\frac{1}{2} g_{\mu \nu} R+\Lambda g_{\mu \nu} I\left(g^{\theta \theta}\right)^{2}=-\frac{8 \pi G}{C^{4}} T_{\mu \nu}$

In this time,

$$
\Lambda=k \frac{G Q^{2}}{C^{4}}, I=\left(\begin{array}{cccc}
1 & 0 & 0 & 0 \\
0 & 1 & 0 & 0 \\
0 & 0 & -1 & 0 \\
0 & 0 & 0 & -1
\end{array}\right)
$$

If $\mathrm{Eq}(1)$ take covariant differential operator,

$$
\begin{aligned}
& \left(R_{\mu \nu}-\frac{1}{2} g_{\mu \nu} R\right)_{i \mu}+\Lambda g_{\mu \nu} I 2 g^{\theta \theta} g^{\theta \theta}{ }_{i \mu}=-\frac{8 \pi G}{C^{4}} T_{\mu \nu ; \mu}=0 \\
& \left(R_{\mu \nu}-\frac{1}{2} g_{\mu \nu} R\right)_{\nu}+\Lambda g_{\mu \nu} I 2 g^{\theta \theta} g^{\theta \theta}=-\frac{8 \pi G}{C^{4}} T_{\mu \nu \nu}=0
\end{aligned}
$$

In this time,

$$
\begin{aligned}
& g_{i \rho}^{\theta \theta}=\frac{\partial g^{\theta \theta}}{\partial x^{\rho}}+2 \Gamma^{\theta}{ }_{\sigma \rho} g^{\sigma \theta}=\frac{\partial g^{\theta \theta}}{\partial r}+2 \Gamma_{\theta x}^{\theta} g^{\theta \theta} \\
& =\frac{\partial}{\partial r}\left(\frac{1}{r^{2}}\right)+2 \cdot \frac{1}{r} \cdot \frac{1}{r^{2}}=0
\end{aligned}
$$

If $g_{i \rho}^{\theta \theta}=V_{\rho}$, the vector transformation is

$$
\mathrm{O}=V_{\rho}=\frac{\partial x^{\alpha}}{\partial x^{\rho}} V_{\alpha}^{\prime}, V_{\alpha}^{\prime}=0
$$

Therefore, if the coordinate is not the spherical coordinate, the covariant differential of $g^{\theta \theta}=\frac{1}{r^{2}}$ is still zero in the changed coordinate 
Unified Theory of Gravity and Electromagnetic Field with Problem in Reissner-Nordstrom and KerrNewman Solution

\section{THE REVISED EINSTEIN GRAVITY EQUATION AND REISSNER-NORDSTROM SOLUTION}

In this theory, $\mathrm{Eq}(1)$ can change the following equation.

$R_{\mu \nu}=-\frac{8 \pi G}{C^{4}}\left(\nabla_{\mu \nu}-\frac{1}{2} g_{\mu \nu} T_{\lambda}^{\lambda}\right)+\Lambda g_{\mu \nu}\left(g^{\theta \theta}\right)^{2}$

In this time, in vacuum, $\mathrm{Eq}(5)$ is

$R_{\mu \nu}=\Lambda g_{\mu \nu} I\left(g^{\theta \theta}\right)^{2}=\Lambda g_{\mu \nu} I \frac{1}{I^{4}}$

Reissner-Nordstrom solution of Einstein-Maxwell equation is

$$
\begin{aligned}
& g_{00}=-1+\frac{2 G M}{r C^{2}}-\frac{k G Q^{2}}{r^{2} C^{4}}, g_{11}=1 /\left(1-\frac{2 G M}{r C^{2}}+\frac{k G Q^{2}}{r^{2} C^{4}}\right) \\
& g_{22}=r^{2}, g_{33}=r^{2} \sin ^{2} \theta
\end{aligned}
$$

The proper time of spherical coordinates is

$d \tau^{2}=A(t, r) d t^{2}-\frac{1}{c^{2}}\left[B(t, r) d r^{2}+r^{2} d \theta^{2}+r^{2} \sin ^{2} \theta d \varphi^{2}\right]$

$g_{\mu \nu} I=\left(\begin{array}{cccc}g_{00} & 0 & 0 & 0 \\ 0 & g_{11} & 0 & 0 \\ 0 & 0 & -g_{22} & 0 \\ 0 & 0 & 0 & -g_{33}\end{array}\right)$

If we use $\mathrm{Eq}(6), \mathrm{Eq}(8)$, we obtain the Ricci-tensor equations.

$$
\begin{aligned}
& R_{t t}=-\frac{A^{\prime} \prime}{2 B}+\frac{A^{\prime} B^{\prime}}{4 B^{2}}-\frac{A^{\prime}}{B r}+\frac{A^{2}}{4 A B}+\frac{\ddot{B}}{2 B}-\frac{\dot{B}^{2}}{4 B^{2}}-\frac{\dot{A} \dot{B}}{4 A B}=-\Lambda A \frac{1}{r^{4}} \\
& R_{r r}=\frac{A^{\prime} \prime}{2 A}-\frac{A^{2}}{4 A^{2}}-\frac{A^{\prime} B^{\prime}}{4 A B}-\frac{B^{\prime}}{B r}-\frac{\ddot{B}}{2 A}+\frac{\dot{A} \dot{B}}{4 A^{2}}+\frac{\dot{B}^{2}}{4 A B}=\Lambda B \frac{1}{r^{4}} \\
& R_{\theta \theta}=-1+\frac{1}{B}-\frac{\not B^{\prime}}{2 B^{2}}+\frac{\not A^{\prime}}{2 A B}=-\Lambda r^{2} \frac{1}{r^{4}}=-\Lambda \frac{1}{r^{2}} \\
& R_{\varphi \varphi}=R_{\theta \theta} \sin ^{2} \theta \\
& R_{t r}=-\frac{\dot{B}}{B r}=0 \\
& R_{t \theta}=R_{t \varphi}=R_{r \theta}=R_{r \varphi}=R_{\theta \varphi}=0
\end{aligned}
$$

In this time, $\quad=\frac{\partial}{\partial r}, \cdot=\frac{1}{c} \frac{\partial}{\partial t}$

If we calculate,

$$
\frac{R_{t t}}{A}+\frac{R_{m}}{B}=-\frac{1}{B r}\left(\frac{A}{A}^{\prime}+\frac{B}{B}\right)^{\prime}=-\frac{(A B)^{\prime}}{n A B^{2}}=0
$$

Hence, we obtain this result.

$$
A=\frac{1}{B}
$$

If $\mathrm{Eq}(16)$ inserts $\mathrm{Eq}(11)$,

$R_{\theta \theta}=-1+\frac{1}{B}-\frac{2 B^{\prime}}{2 B^{2}}+\frac{2 A^{\prime}}{2 A B}=-1+\left(\frac{r}{B}\right)^{\prime}=-\Lambda \frac{1}{r^{2}}$

If we solve $\mathrm{Eq}(17)$,

$$
\begin{aligned}
& \frac{r}{B}=r+C+\frac{\Lambda}{r} \\
& \rightarrow A=\frac{1}{B}=1-\frac{2 G M}{r C^{2}}+\frac{k G Q^{2}}{r^{2} C^{4}}, \Lambda=k \frac{G Q^{2}}{C^{4}}, C=-\frac{2 G M}{C^{2}}
\end{aligned}
$$


Unified Theory of Gravity and Electromagnetic Field with Problem in Reissner-Nordstrom and KerrNewman Solution

Therefore, in vacuum, the spherical solution of the revised Einstein gravity equation is ReissnerNordstrom solution.

$$
d \tau^{2}=\left(1-\frac{2 G M}{r C^{2}}+\frac{k G Q^{2}}{r^{2} C^{4}}\right) d t^{2}-\frac{1}{C^{2}}\left[\frac{d r^{2}}{\left(1-\frac{2 G M}{r C^{2}}+\frac{k G Q^{2}}{r^{2} C^{4}}\right)}+r^{2} d \boldsymbol{\theta}^{2}+r^{2} \sin ^{2} \boldsymbol{\theta} d \boldsymbol{\varphi}^{2}\right]
$$

\section{KerR-Newman Solution and ReviSed Equation With Problem}

In this theory, $\mathrm{Eq}(1)$ can change the following equation.

$\left.R_{\mu \nu}=-\frac{8 \pi G}{C^{4}} \mathbb{T}_{\mu v}-\frac{1}{2} g_{\mu \nu} T_{\lambda}^{\lambda}\right)+\Lambda \operatorname{tg}_{\mu \nu}\left(g^{\theta \theta}\right)^{2}$

In this time, in vacuum, $\mathrm{Eq}(5)$ is

$$
R_{\mu \nu}=\Lambda g_{\mu v} I\left(g^{\theta \theta}\right)^{2}=\Lambda g_{\mu \nu} I \frac{1}{r^{4}}
$$

We think Kerr-Newman equation and solution.

Kerr-Newman solution is

$$
\begin{aligned}
& d s^{2}=g_{00} c^{2} d t^{2}+2 g_{03} c d t d \phi+g_{11} d r^{2}+g_{22} d \theta^{2}+g_{33} d \phi^{2} \\
& I=\left(\begin{array}{cccc}
1 & 0 & 0 & 0 \\
0 & 1 & 0 & 0 \\
0 & 0 & -1 & 0 \\
0 & 0 & 0 & -1
\end{array}\right), g_{\mu \nu} I=\left(\begin{array}{cccc}
g_{00} & 0 & 0 & -g_{03} \\
0 & g_{11} & 0 & 0 \\
0 & 0 & -g_{22} & 0 \\
g_{30} & 0 & 0 & -g_{33}
\end{array}\right), \rho^{2}=r^{2}+a^{2} \cos ^{2} \theta
\end{aligned}
$$

Hence, Kerr-Newman equation is

$$
\begin{aligned}
& R_{t t}=\Lambda g_{00} \frac{1}{\rho^{4}} \\
& R_{r \underline{ }}=\Lambda g_{11} \frac{1}{\rho^{4}} \\
& R_{\theta \theta}=-\Lambda g_{22} \frac{1}{\rho^{4}}=-\Lambda \frac{1}{\rho^{2}} \\
& R_{\phi \phi}=-\Lambda g_{33} \frac{1}{\rho^{4}} \\
& R_{t \phi}=R_{\phi t}=\Lambda g_{03} \frac{1}{\rho^{4}}=\Lambda g_{30} \frac{1}{\rho^{4}} \\
& R_{\phi t}=R_{t \phi}=-\Lambda g_{03} \frac{1}{\rho^{4}}=-\Lambda g_{30} \frac{1}{\rho^{4}}
\end{aligned}
$$

Otherwise $R_{\mu v}=0$

In this time, for proving $\mathrm{Eq}(23)-\mathrm{Eq}(28)$, Maxwell Equation of Einstein-Maxwell equation is $R_{\mu \nu}-\frac{1}{2} g_{\mu \nu} R=-\frac{8 \pi G}{C^{4}} T_{\mu \nu}=-\frac{2 G}{c^{5}}\left(F_{\mu \rho} F_{v}^{\rho}-\frac{1}{4} g_{\mu \nu} F_{\rho \sigma} F^{\rho \sigma}\right)=-\frac{2 G}{C^{5}}\left(g_{\mu \nu} F_{\nu \rho} F^{v \rho}-\frac{1}{4} g_{\mu \nu} F_{\rho \sigma} F^{\rho \sigma}\right)$

In spherical coordinates, if we calculate $\mathrm{Eq}(29)$ of minus term of $g_{03}, g_{30}$ 
Unified Theory of Gravity and Electromagnetic Field with Problem in Reissner-Nordstrom and KerrNewman Solution

$$
\begin{aligned}
& -\frac{2 G}{C^{5}}\left(g_{\mu \nu} F_{\nu \rho} F^{v \rho}-\frac{1}{4} g_{\mu \nu} F_{\rho \sigma} F^{\rho \sigma}\right)=-\frac{2 G}{C^{5}}\left(g_{03} F_{32} F^{32}-\frac{1}{4} g_{03}\left(2 B^{2}-2 E^{2}\right)\right) \\
& =-\frac{2 G}{C^{5}}\left(g_{03} B^{2}-\frac{1}{2} g_{03}\left(B^{2}-E^{2}\right)\right)=-\frac{G}{C^{5}} E^{2} g_{03}=-k \frac{G}{C^{4}} \frac{Q^{2}}{\rho^{4}} g_{03}=-\Lambda g_{03} \frac{1}{\rho^{4}}=-\Lambda g_{30} \frac{1}{\rho^{4}}
\end{aligned}
$$

In spherical coordinates, if we calculate $\mathrm{Eq}(29)$ of plus term of $g_{30}, g_{03}$

$$
\begin{aligned}
& -\frac{2 G}{C^{5}}\left(g_{\mu \nu} F_{\nu \rho} F^{v \rho}-\frac{1}{4} g_{\mu \nu} F_{\rho \sigma} F^{\rho \sigma}\right)=-\frac{2 G}{C^{5}}\left(g_{30} F_{01} F^{01}-\frac{1}{4} g_{30}\left(2 B^{2}-2 E^{2}\right)\right) \\
& =-\frac{2 G}{C^{5}}\left(-g_{30} E^{2}-\frac{1}{2} g_{30}\left(B^{2}-E^{2}\right)\right)=\frac{G}{C^{5}} E^{2} g_{30}=k \frac{G}{C^{4}} \frac{Q^{2}}{\rho^{4}} g_{30}=\Lambda g_{30} \frac{1}{\rho^{4}}=\Lambda g_{03} \frac{1}{\rho^{4}}
\end{aligned}
$$

Hence, Ricci tensor $R_{t \phi}=R_{\phi t}$ have two value term (plus and minus term),

$$
R_{t \phi}= \pm \Lambda g_{03} \frac{1}{\rho^{4}}= \pm \Lambda g_{30} \frac{1}{\rho^{4}}, R_{\phi t}= \pm \Lambda g_{03} \frac{1}{\rho^{4}}= \pm \Lambda g_{30} \frac{1}{\rho^{4}}
$$

The reason of two value term in $\mathrm{Eq}(32)$ is maybe the orientation of rotating (right hand or left hand)..

According to $\mathrm{Eq}(27), \mathrm{Eq}(28)$, we prove $\mathrm{Eq}(30), \mathrm{Eq}(31)$.Therefore, in Kerr-Newman equation and solution, the electromagnetic energy-momentum tensor's formula is equal with the revised Einstein equation's added term of our unified theory. Hence, we know if we solve the revised Einstein's equation, we can obtain Kerr-Newman solution.

\section{CONCLUSiON}

We found the revised Einstein equation of unified theory(the gravity and electromagnetic field).This theory's strong point is 4-dimensional theory. This theory is different from 5-dimensional Kaluza-Klein theory. But as the method of describing universe, Einstein normal gravity equation is equal with the revised Einstein equation of the unified theory because the electric charge has to be zero. .

\section{REFERENCES}

[1] S.Yi, "Spherical Solution of Classical Quantum Gravity", International Journal of Advanced Research in Physical Science, 6,8,2019,pp3-6

[2] S.Yi, "Unified Theory of Gravity and Electromagnetic Field in Reissner-Nodstrom, Kerr-Newman Solution", International Journal of Advanced Research in Physical Science,6,11,2019,pp1-3

[3] S.Yi, "Unification for Gravity and Electromagnetic Field in Kerr-Newman Solution", International Journal of Advanced Research in Physical Science,6,12,2019,pp11-13

[4] S.Weinberg,Gravitation and Cosmology(John wiley \& Sons,Inc,1972)

[5] P.Bergman,Introduction to the Theory of Relativity(Dover Pub. Co.,Inc., New York,1976),Chapter V

[6] C.Misner, K,Thorne and J. Wheeler, Gravitation(W.H.Freedman \& Co.,1973)

[7] S.Hawking and G. Ellis, The Large Scale Structure of Space-Time(Cam-bridge University Press,1973)

[8] R.Adler,M.Bazin and M.Schiffer,Introduction to General Relativity(McGraw-Hill,Inc.,1965)

Citation: Sangwha-Yi, (2020). "Unified Theory of Gravity and Electromagnetic Field with Problem in Reissner-Nordstrom and Kerr-Newman Solution ". International Journal of Advanced Research in Physical Science (IJARPS) 7(1), pp.12-15, 2019.

Copyright: (C) 2020 Authors, This is an open-access article distributed under the terms of the Creative Commons Attribution License, which permits unrestricted use, distribution, and reproduction in any medium, provided the original author and source are credited. 